\title{
Actitudes implícitas en Psicología Ambiental. Una revisión de literatura
}

Jeremías David Tosi. Universidad Nacional de Mar del Plata

Ruben Daniel Ledesma. Universidad Nacional de Mar del Plata

Ariane Kuhnen. Universidade Federal de Santa Catarina

Maíra Longhinotti Felippe. Universidade Federal de Santa Catarina

\section{Resumen}

Este trabajo ofrece una revisión crítica de estudios que utilizan modelos de actitudes implícitas en el área de la Psicología Ambiental. Se analizaron 25 estudios publicados entre 2004 y 2017, los cuales abordan temas relacionados a: (a) conductas proambientales, (b) preferencias por ambientes naturales vs. construidos, (c) conexión con la naturaleza, y (d) cambios en actitudes implícitas. Globalmente, los resultados ilustran las potencialidades de los enfoques de actitudes implícitas en los estudios de Psicología Ambiental. Las actitudes implícitas muestran relaciones de nulas a moderadas con las conductas pro-ambientales. Se observan correlaciones bajas entre actitudes implícitas y explícitas, aunque la relación varía según el dominio. Por otro lado, las medidas de actitudes implícitas tienden a mostrarse incorrelacionadas entre sí. Algunos estudios indican que es posible cambiar las actitudes implícitas. Se identifican limitaciones y posibles líneas futuras de investigación.

Palabras clave: Psicología Ambiental; cognición social; actitudes implícitas.

\section{Resumo}

Atitudes Implícitas em Psicologia Ambiental. Uma Revisão. Este trabalho oferece uma revisão crítica de estudos que utilizam modelos de atitudes implícitas na área da Psicologia Ambiental. Foram analisados 25 estudos publicados entre 2004 e 2017 que abordam temas relacionados a: (a) comportamentos pró-ambientais, (b) preferências por ambientes naturais versus construídos, (c) conexão com a natureza, e (d) mudanças em atitudes implícitas. De modo geral, os resultados ilustram as potencialidades dos enfoques de atitudes implícitas nos estudos da Psicologia Ambiental. Atitudes implícitas mostram correlações nulas a moderadas com comportamentos pro-ambientais. Baixas correlações são observadas entre atitudes implícitas e explícitas, embora a relação varie por domínio. Por outro lado, não há correlações entre diferentes medidas implícitas. Alguns estudos indicam que é possível produzir mudanças em um nível implícito. Limitações e futuras pesquisas são indicadas.

Palavras-chave: Psicologia Ambiental; cognição social; atitudes implícitas.

\section{Abstract}

Implicit Attitudes in Environmental Psychology. A Lietarature Review. This paper provides a review of recent research using implicit attitudes models in Environmental Psychology. Twenty-five studies published between 2004 and 2017 were analyzed. They cover the following topics: (a) pro-environmental behaviors, (b) preferences for natural vs built environments, (c) connection with nature, and (d) changes in implicit attitudes. Overall, the results illustrate the potential of implicit attitudes models and methods in the area of Environmental Psychology. Moderate to null correlations between implicit attitudes and pro-environmental behaviors are observed. Low correlations are reported between implicit and explicit attitudes, although the relationship varies as a function of the object. No correlations between different implicit measures are found. Some studies demonstrate that it is possible to change implicit attitudes. Strengths and limitations of current research are discussed.

Keywords: Environmental Psychology; social cognition; implicit attitudes. 
La Psicología Ambiental ha sido definida como el estudio de las relaciones reciprocas entre fenómenos psicológicos y factores ambientales físicos (Evans, 2005; Gifford, 2014). La investigación en el área adopta un enfoque holístico, donde la persona y el ambiente son concebidas como una unidad que se analiza de forma integrada (Günther, 2005; Günther \& Rozestraten, 1993; Pinheiro, 2003). El principal objetivo del área consiste en mejorar la relación entre el ser humano y su ambiente natural, así como también lograr que los ambientes construidos respondan a las necesidades humanas (Gifford, 2014). Esto involucra diversos temas de investigación, como son la conexión con la naturaleza, la capacidad restauradora de ciertos ambientes, o la preferencia por entornos naturales vs. urbanos (e.g., Gifford, 2014; Günther \& Rozestraten, 1993; Pinheiro, 2003).

A lo largo de su desarrollo la Psicología Ambiental ha recibido importantes aportes teóricos y metodológicos provenientes de la Psicología Social (Günther \& Rozestraten, 1993). En los últimos años, la emergencia y consolidación de modelos que distinguen actitudes implícitas y actitudes explícitas han hecho sentir su influencia en la Psicología Ambiental (e.g., Janpol \& Dilts, 2016; Ratliff, Howell, \& Redford, 2017; Thomas $\&$ Walker, 2016). Entre estos enfoques encontramos, por ejemplo, el Modelo de Actitudes Duales (Wilson, Lindsey, \& Schooler, 2000), el Modelo de Oportunidad y Motivación como Determinantes (Olson \& Fazio, 2009), y el Modelo Asociativo-Proposicional (Gawronski \& Bodenhausen, 2011). Más allá de ciertas diferencias, estos modelos comparten la idea de que las actitudes, como valoraciones positivas o negativas de un objeto dado, existen en dos niveles básicos: explícito e implícito. Por un lado, el nivel explícito involucra evaluaciones basadas en procesos más controlados, concientes y racionales, que implican la validación de creencias. Por otro lado, el nivel implícito se caracteriza por evaluaciones más automáticas, inconcientes e impulsivas, y se encuentra guiado por procesos de naturaleza fundamentalmente asociativa (Blair, Dasgupta, \& Glaser, 2015). Un aspecto interesante de estos modelos es que la conducta final depende de la coexistencia e interacción entre ambos tipos de actitudes, y en ocasiones cada nivel puede motivar cursos diferentes de acción (e.g., Olson \& Fazio, 2009; Strack \& Deutsch, 2004).

Existen dos razones principales que permiten entender la importancia de estos modelos para la Psicología Ambiental. Por un lado, muchos temas se estudian a través de técnicas de auto-informe, como cuestionarios y escalas, que resultan sensibles a distintos tipos de sesgo - como el sesgo de deseabilidad social - (e.g., Ewert \& Galloway, 2004; Sánchez-Miranda \& De la Garza González, 2015). Frente a esta dificultad, los modelos de actitudes implícitas ofrecen alternativas metodológicas de gran interés, denominadas genéricamente "medidas indirectas". Estas medidas, a diferencia de los auto-informes, se basan en procedimientos que permiten reducir ampliamente la posibilidad de control sobre las propias respuestas (Bar-Anan \& Nosek, 2014). Existen evidencias que señalan la capacidad de estos métodos para superar los problemas que afectan a las técnicas de auto-informes, como el sesgo de desabilidad social (Greenwald, Poehlman, Uhlmann, \& Banaji, 2009). Además, el uso combinado de estas nuevas metodologías con las técnicas tradicionales resultaría más congruente con la perspectiva multi-método característica de la Psicología Ambiental (Günther \& Rozestraten, 1993). Por otro lado, la utilidad de los modelos de actitudes implícitas en Psicología Ambiental se relaciona también con la potencialidades de estos enfoques en el estudio de hábitos y comportamientos de naturaleza más automática, como pueden ser las conductas de reciclaje, de consumo energético y de elección de modos de transporte, entre otras (e.g., Geng, Xu, Ye, Zhou, \& Zhou, 2015; Klöckner, 2013). Como sugieren los modelos de actitudes implícitas, en este tipo de conductas, más repetitivas y espontáneas, las actitudes implícitas pueden juegar un rol clave entre los distintos factores explciativos (e.g., Ajzen \& Dasgupta, 2015; Blair et al., 2015).

Este trabajo se propone realizar una revisión de las investigaciones que utilizan modelos de actitudes implícitas en distinos ámbitos de la Psicología Ambiental. Los objetivos específicos son: (a) analizar el tipo de pruebas indirectas que se utilizan para evaluar actitudes implícitas en Psicología Ambiental, (b) identificar las temáticas y conductas que son objeto de estudio, (c) resumir los hallazgos de investigación en cuanto a: la relación entre actitudes implícitas y explícitas, la capacidad predictiva de las actitudes implícitas, y la relación entre distintas medidas indirectas, y (d) identificar limitaciones y posibles líneas futuras de investigación en el área.

\section{Metodología}

Siguiendo las guías PRISMA (Urrutia \& Bonfill, 2013) se realizó una búsqueda de artículos científicos en tres revistas representativas del área y en el buscador Google Scholar durante el mes de enero de 2018 (ver figura 1). Se 
aplicaron búsquedas sucesivas usando diferentes descriptores. Las palabras clave fueron: "actitudes implícitas", "cognición social implícita", "modelo de doble procesamiento", "test de asociaciones implícitas", "psicología ambiental", "conductas ambientales", y "conductas proambientales". Todos los términos de búsqueda se usaron en inglés, portugués, y español. Se incluyeron aquellos trabajos que: (a) estaban publicados en revistas científicas, (b) analizaban temas relacionados con la Psicología Ambiental, y (c) utilizaban medidas indirectas para evaluar actitudes implícitas. En total, se identificaron 25 artículos que cumplían con estos criterios (ver síntesis en tabla 1).
A través de un análisis de contenido se crearon cuatro categorías de agrupación de las investigaciones según similitud temática: (a) conductas pro-ambientales, (b) preferencias por ambientes naturales vs. construidos, (c) conexión con la naturaleza, y (d) cambios en las actitudes implícitas. Mediante este análisis también se identificaron los principales métodos indirectos aplicados en el área. Por lo tanto, para organizar los resultados primero se describen y caracterizan los métodos indirectos utilizados y, posteriormente, se presentan los estudios revisados siguiendo las categorías temáticas mencionadas.

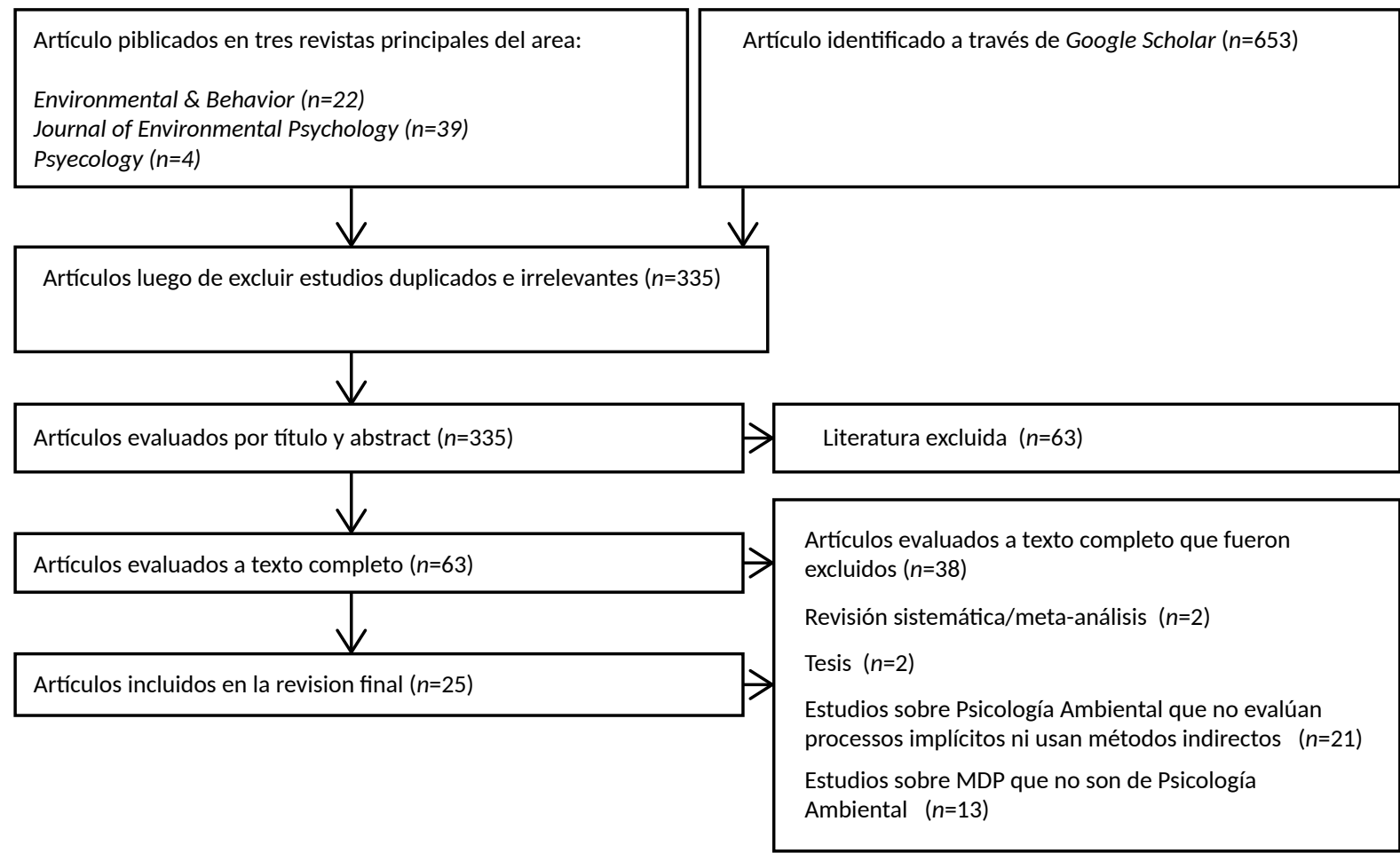

Figura 1. Búsqueda de Literatura.

Tabla 1. Síntesis de los Estudios Revisados sobre MAl en Psicología Ambiental.

\begin{tabular}{|c|c|c|c|c|}
\hline Tema & Autores & Prueba & Constructo & Resultados principales \\
\hline \multirow[t]{4}{*}{$\begin{array}{l}\text { Conductas } \\
\text { pro-ambientales }\end{array}$} & Beattie y Sale (2009) & IAT & $\begin{array}{l}\text { Actitudes hacia productos con } \\
\text { baja huella de carbono }\end{array}$ & Discrepancias entre nivel implícito y explícito \\
\hline & Gamberini et al. (2014) & alAT & $\begin{array}{l}\text { Asociaciones "frases verdaderas } \\
\text { vs. falsas" y ahorro de energía }\end{array}$ & Discrepancias entre nivel implícito y explícito \\
\hline & Ratliff et al. (2017) & SR & $\begin{array}{l}\text { Actitudes hacia el prototipo } \\
\text { ambientalista }\end{array}$ & $\begin{array}{l}\text {-Actitudes implícitas predicen conductas pro-ambientales } \\
\text {-Correlación moderada-alta entre lo implícito y explícito }\end{array}$ \\
\hline & Thomas y Walker (2016) & IAT & $\begin{array}{l}\text { Preferencias por valores } \\
\text { biosfericos vs egoístas }\end{array}$ & $\begin{array}{l}\text {-IAT: (a) discrimina grupos de ambientalistas/no- } \\
\text { ambientalistas; (a) predice status ambientalista } \\
\text {-Correlación baja-moderada entre lo implícito y explícito }\end{array}$ \\
\hline
\end{tabular}


Tabla 1. Continuación

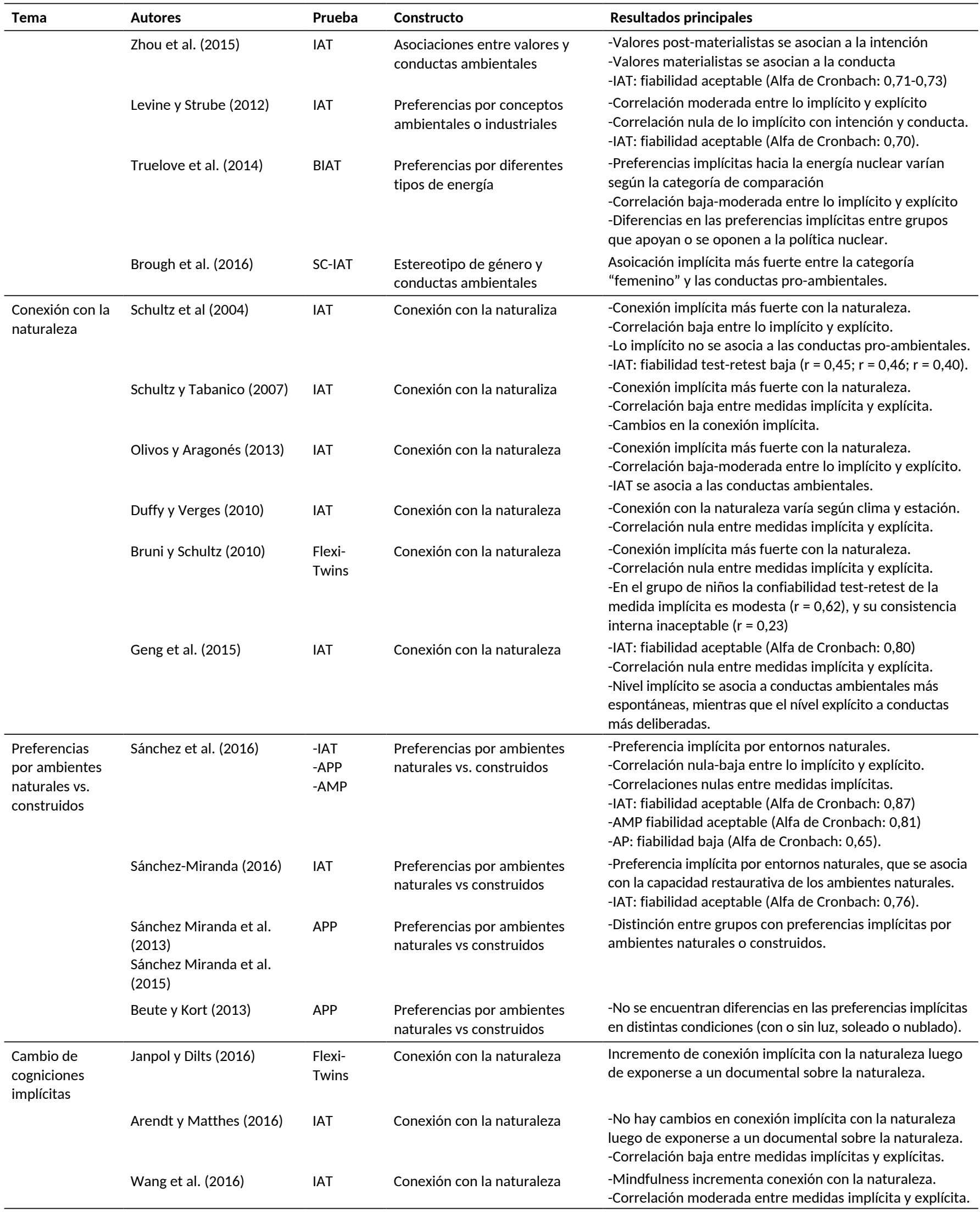


Actitudes implícitas en psicología ambiental. Una revisión de literatura

Tabla 1. Continuación

\begin{tabular}{|c|c|c|c|c|}
\hline Tema & Autores & Prueba & Constructo & Resultados principales \\
\hline & Geng et al. (2013) & IAT & Actitudes hacia el reciclaje & $\begin{array}{l}\text {-Condicionamiento evaluativo produce cambios en } \\
\text { actitudes implícitas hacia el reciclaje. }\end{array}$ \\
\hline & Tate et al. (2014) & IAT & $\begin{array}{l}\text { Actitudes implícitas hacia el } \\
\text { reciclaje }\end{array}$ & $\begin{array}{l}\text {-Mensajes pro-ambientales aumentan actitudes implícitas } \\
\text { y favorecen elecciones de consumo más sustentables. }\end{array}$ \\
\hline & Bruni et al. (2015) & IAT & Conexión con la naturaleza & $\begin{array}{l}\text {-Conexión implícita más fuerte con la naturaleza, } \\
\text { independientemente de la valencia de los estímulos } \\
\text { utilizados en el IAT. }\end{array}$ \\
\hline
\end{tabular}

\section{Resultados}

Medidas Indirectas Utilizadas en el Área

En los estudios revisados se observa el uso de diferentes pruebas indirectas, como son el Implicit Association Test (IAT) (Greenwald, McGhee, \& Schwartz, 1998), el Single Category-IAT (SC-IAT) (Karpinski \& Steinman, 2006), o el Affective Misatribution Procedure (AMP) (Payne, Cheng, Govorun, \& Stewart, 2005). Sin embargo, el IAT es la medida más utilizada (17/25 artículos). Esto es esperable si se considera que esta prueba posee una mayor difusión y popularidad que el resto (Bar-Anan \& Nosek, 2014). EI IAT es una tarea basada en tiempos de respuesta que evalúa el grado de asociación entre dos categorías de atributos (e.g., "bueno vs. malo") y dos categorías target. Por ejemplo, si se evalúan las actitudes implícitas hacia ambientes naturales (vs. ambientes urbanos) las categorías target podrían ser "ciudad vs. naturaleza" (e.g., Sánchez-Miranda, 2016). Durante la tarea la persona debe clasificar los estímulos de las cuatro categorías utilizando dos opciones de respuesta, en dos condiciones opuestas denominadas bloque compatible e incompatible. El puntaje final surge de la diferencia en las latencias de respuesta en estas dos condiciones, y representa la fuerza de asociación "implícita" entre las categorías de los atributos y los target. Es importante señalar que el IAT ha demostrado ser una medida más robusta que las técnicas tradicionales frente a la deseabilidad social (Greenwald et al., 2009), y ha exhibido propiedades psicométricas superiores a otras pruebas indirectas (Bar-Anan \& Nosek, 2014).

Los estudios en el área también han utilizado algunas variantes del IAT. Dos de estas pruebas varían específicamente en cuanto a la cantidad de categorías target. Por un lado, el Brief Implicit Association Test (BIAT) (Sriram \& Greenwald, 2009) permite comparar las asociaciones entre dos categorías de atributos y cuatro categorías target. Así, el único estudio del área que utilizó el BIAT midió las preferencias por cuatro formas de energía (i.e. nuclear, eólica, carbón y gas natural) (Truelove, Greenberg, \& Powers, 2014). Por otro lado, el SC-IAT se centra en una sola categoría target. Por ejemplo, Brough, Wilkie, Ma, Isaac y Gal (2016) desarrollaron dos versiones del SC-IAT para medir el estereotipo implícito basado en la asociación entre el género y las conductas pro-ambientales. En una versión la categoría target era el género "femenino", mientras que en otra era el "masculino". Es importante destacar que, a diferencia del IAT y el BIAT, el SC-IAT permite obtener un resultado "absoluto" (i.e. asociaciones implícitas entre los atributos y una sola categoría target) en vez de "relativo" (i.e. asociaciones implícitas entre los atributos y dos o cuatro categorías target). Por otra parte, un aspecto importante del BIAT y el SC-IAT es que ambas pruebas mostraron buenas propiedades psicométricas (Bar-Anan \& Nosek, 2014).

Otro estudio del área utilizó una variante del IAT denominada Autobiographical Implicit Association Test (Sartori, Agosta, Zogmaister, Ferrara, \& Castiello, 2008). En esta prueba se usan cuatro categorías: dos pertencientes a "oraciones verdaderas vs. falsas" y dos que representan eventos autobiográficos opuestos. En el estudio que utilizó el alAT estos eventos aludían a la "conducta de ahorrar vs. desperdiciar energía" (Gamberini et al., 2014). El supuesto del alAT es que la fuerza de la asociación entre estos eventos y las oraciones verdaderas o falsas permite conocer cuál de los dos eventos es "verdadero". En este sentido, esta medida actuaría como un "detector de engaño" (lie detector). Sin embargo, el uso del alAT en este sentido resulta problemático, pues la evidencia indica esta medida es vulnerable a la manipulación de las respuestas cuando la persona está familiarizada con la tarea (ver Suchotzki, Verschuere, Van Bockstaele, Ben-Shakhar, \& Crombez, 2017).

También existen investigaciones que usaron pruebas indirectas de priming evaluativo para medir actitudes implícitas hacia diferentes tipos de ambiente. En esta línea, Sánchez-Miranda et al. (2013) aplicaron una 
medida basada en el Affective Priming Paradigm (APP) que consiste en la presentación rápida de un estímulo facilitador o prime (en este caso perteneciente a las categorías "ciudad vs. naturaleza"), seguido de un estímulo que corresponde a las categorías de los atributos semánticos o valorativos (e.g., "bueno vs. malo"). En esta tarea se espera que el estímulo prime facilite o inhiba la clasificación del segundo estímulo. Por su parte, Sánchez et al. (2016) utilizaron el AMP, el cual se basa en la tendecia a atribuir una respuesta afectiva generada por un estímulo (e.g., ambiente natural) a otro estímulo ambiguo (e.g., letra china). En esta prueba el estímulo facilitador que activa la reacción afectiva es seguido por una letra china y la persona clasifica esta última como agradable o desagradable. Es importante señalar que entre las técnicas de priming mencionadas el AMP ha mostrado cualidades psicométricas claramente superiores al APP (ver Bar-Anan $\&$ Nosek, 2014). Esto puede puede advertirse en un estudio del área, específicamente en relación a la consistencia interna de las dos medidas (Sánchez et al., 2016). En consecuencia, entre las pruebas de priming evaluativo el AMP parece una alternativa más recomendable.

Por último, un estudio del área utilizó el Speeded Self-report (SR) para evaluar las actitudes implícitas hacia el prototipo ambientalista (Ratliff et al., 2017). Esta prueba consiste en clasificar rápidamente estímulos que representan el objeto de actitud en una escala de 1 a 4 según diferentes adjetivos. En el estudio mencionado el objeto fue el "prototipo ambientalista" y los adjetivos fueron "atractivo", "cool", "divertido", "inteligente" y "juicioso". Un problema central de esta prueba es que no está claro si se trata de una medida directa o indirecta. Si bien Ratliff et al. (2017) asumen que el SR es una medida indirecta por el hecho de implicar presión de tiempo, el procedimiento básico es muy semejante al que se utiliza en auto-informe tradicional. Por esta razón, algunos autores lo clasifican como una medida directa (e.g. Bar-Anan \& Nosek, 2014).

\section{Conductas Pro-Ambientales y Cogniciones Implícitas}

Las conductas pro-ambientales involucran un conjunto de comportamientos que tienen un impacto positivo -o el menor impacto negativo posible- en el ambiente (e.g., reducción de residuos, ahorro de energía, uso de transporte activo) (e.g., Stern, 2000). En los últimos años estos comportamientos han sido el foco de numerosas investigaciones, debido en gran medida a una creciente preocupación en materia ambiental (Morren \& Grinstein, 2016; Turaga, Howarth, $\&$ Borsuk, 2010). Para conocer los factores psicológicos asociados a estas conductas, varios autores han recurrido a los modelos de actitudes implícitas.

Es el caso de Leviney Strube (2012), quienes estudiaron las preferencias implícitas por conceptos "ambientales" (e.g., reciclaje) o "industriales" (e.g., polución) y hallaron que éstas se asociaban con las preferencias explícitas, pero no con la intención ni con las conductas pro-ambientales. Según los modelos de actitudes implícitas, la ausencia de relación entre el nivel implícito y la intención ocurre porque las actitudes implícitas se caracterizan por ser menos intencionales (Gawronski \& Bodenhausen, 2011). Por otro lado, la falta relación entre lo implícito y la conducta es una cuestión más difícil de explicar. En esta investigación un elemento a tener en cuenta es que la medida implícita estaba conformada por categorías que no representaban la conducta en sí misma, sino que correspondían a conceptos ambientales generales.

En otro estudio Truelove et al. (2014) analizaron las preferencias implícitas por cuatro tipos de energía: nuclear, eólica, carbón y gas natural. Los resultados exhibieron preferencias implícitas negativas hacia la energía nuclear cuando se comparaba con el gas natural y la energía eólica, y preferencias positivas cuando la categoría opuesta era el carbón. También se encontraron correlaciones de bajas a moderadas entre el nivel implícito y explícito. Finalmente, se observaron diferencias en las preferencias implícitas entre grupos que se oponen o apoyan la política nuclear: los primeros prefieren el gas natural (vs. energía nuclear), mientras que los segundos prefieren la energía nuclear (vs. carbón).

Otras investigaciones han utilizado los modelos de actitudes implícitas para estudiar valores relacionados con conductas ambientales. Por ejemplo, Thomas y Walker (2016) midieron la preferencia implícita por valores biosfericos vs. egoístas, y ofrecieron tres resultados centrales. Primero, se encontraron correlaciones de bajas a moderadas entre las preferencias implícitas y las escalas explícitas de valores. Segundo, el IAT fue capaz de discriminar entre grupos de ambientalistas y no-ambientalistas (i.e. los primeros mostraron preferencias más fuertes por valores biosfericos) con tamaños del efecto de medianos a grandes. Por último, el IAT fue la variable de mayor importancia en la predicción del status ambientalista de los participantes.

También Zhou, Ye, Geng y Xu (2015) analizaron las asociaciones implícitas entre valores materialistas o post-materialistas y las conductas ambientales. Mientras que la dimensión post-materialista se asoció de forma 
significativa con la intención de llevar adelante conductas pro-ambientales, los valores materialistas fueron predictores significativos de la conducta pro-ambiental actual (i.e. cantidad de papel gastado en una tarea propuesta por los investigadores). Según los autores, los resultados hallados se entienden porque tanto el comportamiento estudiado como los valores materialistas se basan en procesos más impulsivos, mientras que la intención evaluada a través de un auto-informe y los valores post-materialistas depende en mayor medida de procesos reflexivos. De todos modos, es importante señalar que la forma de medir la conducta actual en este estudio no resulta representativa de los comportamientos pro-ambientales en toda su variedad y complejidad. Este es un problema que los mismos autores identifican entre las limitaciones del estudio.

Continuando con otros antecedentes, Ratliff et al. (2017) estudiaron las actitudes implícitas hacia el prototipo ambientalista mediante la prueba SR. Los resultados mostraron actitudes implícitas menos favorables hacia el prototipo ambientalista, en comparación con las actitudes explícitas. Asimismo, las actitudes implícitas y explícitas se relacionaron positivamente con la frecuencia informada de conductas pro-ambientales, aunque el peso de lo implícito fue mayor. También se observó que cuando las actitudes implícitas eran más positivas aumentaba la probabilidad de realizar donaciones a organizaciones ambientales. Por el contrario, no se observó ninguna asociación cuando la organización tenía otros fines (e.g., proteger animales), lo que podría entenderse como evidencia de validez discriminante. Por último, se encontraron correlaciones de moderadas a altas entre actitudes implícitas y explícitas, las cuales podrían deberse a la similitud estructural entre el SR y la prueba directa.

Finalmente, se identificaron dos investigaciones que analizan exclusivamente la relación entre medidas implícitas y explícitas. Por un lado, Beattie y Sale (2009) estudiaron las actitudes implícitas hacia productos con baja huella de carbono e identificaron un subgrupo de participantes que presentaba una proporción mayor de actitudes explícitas positivas, en comparación con la proporción de actitudes implícitas positivas. Por otro lado, Gamberini et al. (2014) estudiaron conductas de ahorro de energía a través del alAT. Como parte de los resultados se encontró que un segmento de los participantes declaró de forma explícita realizar conductas de ahorro de energía, pero en la prueba indirecta presentó "memorias contradictorias" (i.e. la conducta se asoció en menor medida con frases verdaderas).
Los dos estudios anteriores concluyeron que las diferencias halladas constituyen un indicador de la discrepancia entre cogniciones implícitas y explícitas, la cual sería producto de los efectos de deseabilidad social sobre la medida explícita. Si bien se trata de un resultado interesante, las inferencias planteadas presentan una limitación importante. El análisis de la relación implícito-explícito está basado en porcentajes directos (no estandarizados) provenientes de escalas que poseen un cero arbitrario. Cabe destacar que esta métrica arbitraria se encuentra tanto en las medidas directas como en las indirectas (e.g., Blanton \& Jaccard, 2006). Esto significa, por ejemplo, que las actitudes implícitas positivas por productos con baja huella de carbono no necesariamente son equivalentes a las actitudes explícitas positivas por el mismo objeto. Por lo tanto, sería correcto recurrir a otros indicadores estadísticos para conocer el tipo de relación entre ambas actitudes (e.g., correlación de Pearson). Además, si se aceptara la supuesta discrepancia, sería un error proponer que únicamente la deseabilidad social es capaz de moderar la relación. Por el contrario, es posible la participación de múltiples factores moderadores más allá del deseabilidad social (e.g., Nosek, 2005).

En síntesis, la investigación desde los modelos de actitudes implícitas permite advertir que las actitudes contribuyen a una mejor comprensión de los comportamientos pro-ambientales. En algunos casos, se observa además que el peso relativo del componente implícito sería superior al explícito (Ratliff et al., 2017). Por otra parte, todos los estudios muestran un grado de convergencia de bajo a moderado entre actitudes implícitas y explícitas, el cual es consistente con los resultados encontrados en el área de los modelos de actitudes implícitas. Un aspecto que no fue abordado en ningún trabajo corresponde al estudio de factores que moderan la relación implícito-explícito. Finalmente, se han sugerido algunas limitaciones que, en general, indican la necesidad de ser cautelosos y críticos al momento de interpretar los resultados de algunos estudios.

\section{Preferencias por Ambientes Naturales vs Construidos}

Dentro de la Psicología Ambiental también se ha estudiado la preferencia implícita por ambientes naturales o construidos. Su medición se ha realizado a través de diferentes métodos indirectos, incluyendo el IAT, el APP, y el AMP. A pesar de poseer algunas diferencias estructurales, estas pruebas mantienen las mismas categorías de asociación para evaluar preferencias 
implícitas por diferentes entornos: "ciudad vs. naturaleza", y "bueno vs. malo".

La evidencia empírica sobre preferencias implícitas por diferentes tipos de ambiente presenta resultados que no son concluyentes. Sánchez-Miranda (2016) ofrece información a favor de una preferencia implícita por los entornos naturales, la cual se asocia con la percepción explícita de que estos ambientes "restauran" el bienestar psicológico, físico y social. Por su parte, Sánchez-Miranda, Garza González y Isolde Hedlefs (2016) usaron tres medidas indirectas diferentes que indicaron el mismo resultado: una preferencia implícita por los ambientes naturales. Un aspecto a considerar de este estudio es que las tres pruebas indirectas no se relacionaron entre sí (la relación implícito-implícito es un tema controvertido que se retomará de forma más detallada en la discusión). Además, solamente una de ellas (i.e. AMP) se asoció con las preferencias explícitas, lo que podría deberse a similitudes estructurales con la escala explícita que no se presentan en el resto de las pruebas indirectas (i.e. IAT y tarea de priming afectivo). Como se sabe, la estructura de los métodos implícitos y explícitos incide en la fuerza de la relación (Payne, Burkley, \& Stokes, 2008).

Otros estudios han cuestionado las evidencias previamente señaladas, mostrando que no todas las personas presentan una preferencia implícita por los entornos naturales. En este sentido, se han distinguido dos grupos de personas: aquellas que se denominan "verdes" debido a sus preferencias implícitas por la naturaleza, y quienes se clasifican como "urbanos" dado que prefieren implícitamente entornos urbanos (e.g., Sánchez-Miranda \& De la Garza González, 2015; Sánchez-Miranda, De la Garza González, \& Rangel Sandoval, 2013). Por su parte, Beute y Kort (2013) encontraron preferencias explícitas, aunque no implícitas, en favor de los entornos naturales, luminosos (vs. sín luz) y soleados (vs. nublados).

En resumen, los modelos de actitudes implícitas enriquecen el estudio de las preferencias por diferentes tipos de ambientes, ofreciendo nuevos conceptos y herramientas que permiten profundizar el debate. A diferencias de la amplia literatura sobre preferencias explícitas, el estudio de las preferencias implícitas es menor y los resultados no son consistentes. Un aspecto que ha recibido poca atención en los estudios citados involucra el análisis de la relación entre las preferencias implícitas y explícitas, así como también entre lo implícito y la conducta ambiental.

\section{Conexión con la Naturaleza}

La noción de conexión con la naturaleza constituye un constructo psicológico específico del área que también ha sido analizado a través de pruebas indirectas. El concepto se refiere al grado en que la persona se percibe como parte del ambiente natural (e.g., Bruni, Chance, Schultz, \& Nolan, 2012). A nivel implícito esto supone una asociación automática entre conceptos relacionados con uno mismo y los entornos naturales, en comparación con los construidos. Por esta razón, para medirlo se ha utilizado una versión del IAT que originalmente evalúa autoconcepto (Greenwald \& Farnham, 2000). En esta versión las categorías de asociación son las siguientes: yo (e.g., mío, mi), otro (e.g., ellos, su), ambiente natural (e.g., plantas, animales), y ambiente construido (e.g., ciudad, edificios).

Una discusión que se presenta en la mayoría de los estudios alude a la relación entre la conexión implícita con la naturaleza y la hipótesis de biofilia (e.g., Schultz, 2004). Según esta hipótesis, existe una disposición innata, universal y relativamente estable a conectarse con la naturaleza. En consonancia, las investigaciones deberían mostrar una asociación implícita "yo-ambiente natural" más fuerte, en comparación con la asociación "yo-ambiente construido". Sin embargo, la evidencia empírica no es clara. Si bien la mayoría de los trabajos apoyan la hipótesis de biofilia (Bruni et al., 2012; Olivos \& Aragonés, 2013; Schultz, Shriver, Tabanico, \& Khazian, 2004; Schultz \& Tabanico, 2007), existen estudios que cuestionan la presunta estabilidad de la conexión implícita con la naturaleza. En particular, Duffy y Verges (2010) identificaron fluctuaciones en la asociación "yo-ambiente natural" en función de cambios meteorológicos y estacionales (i.e. mayor conexión implícita en días soleados y durante la primavera o en otoño, en comparación con los días de lluvia y en invierno).

En cuanto a la relación entre el IAT y varias medidas explícitas (i.e. escala de conexión con la naturaleza y dos escalas que evalúan valores biosfericos y altruistas) se han encontrado correlaciones nulas (Duffy \& Verges, 2010; Geng et al., 2015) o bajas (Olivos \& Aragonés, 2013; Schultz et al., 2004; Schultz $\&$ Tabanico, 2007). Por su parte, la relación entre el nivel implícito y la conducta también presenta evidencias contrapuestas: mientras que en Olivos y Aragonés (2013) la medida implícita se asoció levemente con una escala de comportamientos ambientales, en Schultz et al. (2004) no se halló una relación significativa. En este último aspecto, es interesante destacar los aportes de Geng et al. (2015), quienes mostraron que la conexión 
implícita y explícita con la naturaleza predice distintos tipos de comportamiento ambientales: mientras que la primera se relaciona con conductas más espontáneas, la segunda se asocia con conductas más deliberadas. Esta evidencia es consistente con uno de los supuestos principales de los modelos de actitudes implícitas (e.g., Greenwald et al., 2009).

Finalmente, Bruni y Schultz (2010) desarrollaron una versión del IAT denominada FlexiTwins con el objetivo de evaluar la conexión implícita con la naturaleza en niños. La prueba conserva la estructura tradicional del IAT, pero se presenta como un juego en una plataforma colorida y animada, donde se añaden sonidos y acumulación de puntos para dar una retroalimentación a las respuestas. Para validar esta medida se utilizaron tres muestras diferentes: estudiantes universitarios, activistas ambientales, y niños. Los resultados permitieron ver que la mayoría de los participantes tenían una asociación implícita más fuerte con la naturaleza. En el caso de los niños, esta conexión implícita disminuía cuando los niños informaban destinar más tiempo a actividades de ocio dentro de la casa (e.g., mirar televisión o jugar videojuegos). Por otra parte, mientras que en estudiantes universitarios se hallaron patrones de correlación moderados entre la medida implícita y las explícitas (i.e. escalas de conexión con la naturaleza, intención de conductas ambientales y preocupaciones ambientales), en las dos muestras restantes el nivel implícito no se asoció con las escalas explícitas. La falta de relaciones en niños y activistas podría atribuirse principalmente al hecho de que las medidas explícitas estaban destinadas a población general, es decir que no fueron adaptadas a grupos específicos como los mencionados.

En resumen, los modelos de actitudes implícitas permiten comprender aspectos menos concientes de la conexión con la naturaleza, y amplían la discusión de otros aspectos conceptuales vinculados con este fenómeno (e.g., la hipótesis de biofilia). Nuevamente, un tema que merece mayor atención corresponde al rol de la conexión implícita por la naturaleza en la comprensión de las conductas ambientales.

\section{Cambio en las Actitudes Implícitas}

Un área interesante comprende el estudio sobre la maleabilidad de las actitudes implícitas, es decir, la posibilidad de que estas puedan cambiar o ser modificadas. A pesar de que las actitudes implíctas se suponen más estables y resistentes (e.g., Rydell \& McConnell, 2006), existe evidencia sobre cierta permeabilidad al cambio. Por ejemplo, Schultz y Tabanico (2007), encontraron un incremento de la conexión implícita con la naturaleza luego de visitar espacios naturales (e.g., la playa). Algo importante es que estos cambios se lograban solo cuando la exposición era más extendida en el tiempo (vs. una exposición de cinco minutos). En la misma línea, otro estudio mostró una mayor asociación "yo-naturaleza" y un aumento en la probabilidad de donar dinero a organizaciones ambientales, después de exponer a los participantes a un documental centrado en entornos naturales (vs. un documental sobre entornos urbanos) (Janpol \& Dilts, 2016).

En otras investigaciones también se obtuvieron cambios a nivel implícito a través de mindfulness, condicionamiento evaluativo, y mensajes pro-ambientales. Primero, Wang et al. (2016) compararon el uso de "mindfulness" vs. "mindlessness" y encontraron que en la primera condición la conexión implícita y explícita hacia la naturaleza era superior. En segundo lugar, Geng, Liu, Xu, Zhou y Fang (2013) analizaron si el condicionamiento evaluativo producía cambios en las actitudes implícitas hacia el reciclaje. El procedimiento consistió en emparejar imágenes que aludían al reciclaje con estímulos placenteros e imágenes que representaban el consumismo con estímulos displacenteros. Por medio de esta técnica se incrementaron las actitudes implícitas positivas hacia el reciclaje. Finalmente, se ha observado que el uso de mensajes pro-ambientales promueve actitudes implícitas más positivas hacia productos comestibles no envasados y favorece elecciones de consumo más sustentables, pero no genera cambios a nivel explícito (Tate, Stewart, \& Daly, 2014).

Por otro lado, Arendt y Matthes (2016) estudiaron los efectos de la exposición a un documental sobre entornos naturales vs. no naturales, pero no encontraron diferencias en la conexión implícita con la naturaleza. En este caso, la intervención propuesta tenía una duración bastante acotada (i.e. 7 minutos), es decir, era más breve en comparación con los estudios mencionados. En este sentido, es posible que la brevedad de la exposición explique la ausencia de efecto. Como sugieren investigaciones en otros dominios (Rydell \& McConnell, 2006), la necesidad de aumentar la cantidad y el tiempo de exposición para obtener cambios se encuentra respaldada por el hecho de que las actitudes implícitas se desarrollan a través de aprendizajes más lentos.

En síntesis, la evidencia actual sobre la maleabilidad de las las actitudes implícitas en Psicología Ambiental muestra que existen caminos posibles para producir cambios a este nivel. Un punto de interés 
escasamente considerado corresponde a la comparación de los cambios obtenidos a nivel implícito y explícito. La posibilidad de generar conocimiento sobre esta cuestión podría tener un impacto práctico, dado que permitiría distinguir cuáles son las estrategias útiles para modificar actitudes implícitas y/o explícitas. No obstante, se trata de una cuestión donde la investigación todavía es incipiente.

\section{Discusión}

Este trabajo ofreció una revisión de estudios que aplican modelos de actitudes implícitas al campo de la Psicología Ambiental. La literatura relevada muestra cómo, en diversos temas, la investigación puede verse enriquecida por estos enfoques. El uso de las pruebas indirectas, principalmente el IAT en sus diversas versiones, amplía las alternativas técnicas disponibles y permite conocer actitudes que no logran captarse a través de los instrumentos tradicionales. A su vez, la aplicación de los modelos de actitudes implícitas ha generado nuevas preguntas y desafíos que incentivan el desarrollo de más investigación.

Una cuestión importante corresponde al estudio de las actitudes implícitas en la predicción de las conductas pro-ambientales, las cuales ocupan un lugar prioritario entre las líneas actuales de la Psicología Ambiental (e.g., Moser, 2003). En este sentido, los resultados de los estudios revisados no son consistentes, pues se observan correlaciones de nulas a moderadas (Levine \& Strube, 2012; Olivos \& Aragonés, 2013; Schultz et al., 2004; Zhou et al., 2015). A su vez, un solo estudio muestra que las medidas implícitas incrementan la predicción de las conductas pro-ambientales con respecto a las actitudes explícitas (Ratliff et al., 2017). Entendemos que la variabilidad de estos resultados refleja en gran medida las diferencias existentes en cuanto a los constructos, conductas y metodologías utilizadas en los distintos estudios. Una primera cuestión es que los estudios evalúan en rigor distintos contructos, algunos de los cuales estarían más próximos a la valoración de las conductas que se buscan predecir (e.g., actitudes), mientras que otros serían más distales (e.g., conexión con la naturaleza). Asimismo, las conductas son muy variables, pues algunos estudios utilizan puntajes totales de escalas que contienen un conjunto diverso de conductas ambientales, mientras que otros analizan conductas específicas (e.g., uso de bolsas de plástico o uso de papel). Es importante señalar que, en el último caso mencionado, la relación con lo implícito tiende a ser más fuerte. En síntesis, se requiere más investigación para clarificar la importancia relativa de las actitudes implícitas en las conductas ambientales, así como mayor profundidad en el estudio de posibles factores moderadores de esta relación.

Otro asunto importante es el grado de relación o convergencia entre actitudes implícitas y explícitas. Nuevamente, las investigaciones en el área muestran resultados muy diversos, con correlaciones que van de nulas a fuertes (e.g., Arendt \& Matthes, 2016; Thomas $\&$ Walker, 2016). En este caso, la fuerza de las relaciones varía en función del tema estudiado. Los trabajos centrados en la conexión con la naturaleza presentan correlaciones más bajas, en comparación con aquellos que analizan preferencias y actitudes. Este resultado coincide con la literatura previa disponible en otras áreas en cuanto a la existencia de variaciones en la relación entre medidas implícitas y explícitas de acuerdo al dominio estudiado (Bar-Anan \& Nosek, 2014). No obstante, el estudio de los moderadores de la relación implícito-explícito requeriría investigaciones centradas exclusivamente en esta cuestión y fundamentadas en conceptualizaciones específicas. Por ejemplo, Nosek (2005) sugiere un esquema de cuatro moderadores principales que han resultado relevantes en algunos dominios (e.g., prejuicios, preferencias políticas, preferencias por distintos grupos de edad, actitudes hacia conductas relacionas con el consumo de alcohol). Esta línea de investigación no ha sido explorada en Psicología Ambiental.

La última cuestión a destacar es la relación entre distintas pruebas indirectas. Este es un asunto fundamental, pues remite a problemas de validez y fiabilidad de las pruebas, y al grado en que estas estarían evaluando realmente los mismos procesos implicados en las actitudes implícitas. El único estudio que analizó este tema arrojó resultados que alertan sobre posibles problemas de validez, informando correlaciones próximas a cero entre tres pruebas indirectas (Sánchez et al., 2016). En general, estudios previos han observado correlaciones bajas entre disitntas prubeas indirectas (Bar-Anan \& Nosek, 2014), lo que suele atribuirse a tres factores. Un primer factor se relaciona con la fiabilidad de los métodos indirectos (e.g., Nosek, Greenwald, \& Banaji, 2007). Algunas medidas implícitas, como las tareas de priming, presentan niveles de confiabilidad bajos, afectando de este modo las estimaciones de correlación. De hecho, las medidas que tienen buena confiabilidad (e.g. IAT o AMP) tienden a mostrar correlaciones más altas entre sí (Bar-Anan \& Nosek, 2014; 
Gawronski \& Hahn, 2017). Un segundo factor se relaciona con la existencia de diferencias importantes entre las tareas a nivel procedimental, lo que podría dar lugar a la medición de procesos que realmente son distintos (Payne et al., 2008). Se ha estudiado que la fuerza de la relación aumenta en la medida en que las pruebas presentan mayor similitud operacional (Bar-Anan \& Nosek, 2014, Gawronski \& Hahn, 2017). El problema aquí no es solo el grado de similitud "operativa", sino la diversidad de características asociadas a las actitudes implícitas (i.e. conciencia, eficiencia, control e intencionalidad). De aquí surge el tercer factor, donde se plantea que cada prueba indirecta mide diferentes características del nivel implícito (De Houwer et al., 2009; Gawronski \& Hahn, 2017). En cualquier caso, las medidas indirectas enfrentan el desafío de mejorar su fiabilidad, aclarar posibles diferencias procedimentales e identificar el tipo de proceso implícito que logran retener (e.g, menor control y/o mayor eficiencia).

Es necesario mencionar que nuestra revisión presenta como limitación principal la metodología utilizada para identificar los estudios de interés. Por un lado, si bien se utilizaron descriptores que intentaban representar la temática estudiada, es posible que no hayan sido considerados otros descriptores que también podrían ser útiles. Por otro lado, una de las fases de búsqueda consistió en la lectura del título, el resumen y las palabras clave, pero no del artículo completo. Asimismo, solo fueron incluidos en la búsqueda trabajos en idioma portugués, español e inglés. En consecuencia, debido a estas decisiones metodológicas es posible que existan artículos pertinentes pero que no hayan sido detectados en la búsqueda.

En síntesis, los resultados de la investigación aún resultan inconsistentes en varios aspectos centrales para el estúdio de las actitudes implícitas y su relación con las conductas ambientales. Por lo tanto, se requiere profundizar la aplicación de los modelos de actitudes implícitas a través de investigaciones que superen las limitaciones de los estudios previos. Entre otras cosas, será necesario optimizar la validez y confiabilidad de los métodos indirectos, abordar conductas ambientales más específicas, y analizar los posibles moderadores de la relación entre actitudes implícitas y explícitas.

\section{Referencias}

Ajzen, I., \& Dasgupta, N. (2015). Explicit and implicit beliefs, attitudes, and intentions: The role of conscious and unconscious processes in human behavior. En P. Haggard \& B. Eitam (Orgs.), The Sense of Agency (pp. 115-144). Nueva York: Oxford University Press. doi: 10.1093/acprof:oso/9780190267278.003.0005

Arendt, F., \& Matthes, J. (2016). Nature documentaries, connectedness to nature, and pro-environmental behavior. Environmental Communication, 10(4), 453-472. doi: 10.1080/17524032.2014.993415

Bar-Anan, Y., \& Nosek, B. A. (2014). A comparative investigation of seven indirect attitude measures. Behavior Research Methods, 46, 668-688. doi: 10.3758/s13428-013-0410-6

Beattie, G., \& Sale, L. (2009). Explicit and implicit attitudes to low and high carbon footprint products. International Journal of Environmental, Cultural, Economic and Social Sustainability, 5(4), 191-206. doi: 10.18848/1832-2077/CGP/v05i04/54652

Beute, F., \& Kort, Y. A. W. (2013). Let the sun shine! Measuring explicit and implicit preference for environments differing in naturalness, weather type and brightness. Journal of Environmental Psychology, 36, 162-178. doi: 10.1016/j.jenvp.2013.07.016

Blair, I. V., Dasgupta, N., \& Glaser, J. (2015). Implicit attitudes. En M. E. Mikulincer, P. R. Shaver, J. F. Dovidio, \& J. A. Simpson (Orgs.), Handbook of personality and social Psychology, Volume 1: Attitudes and social cognition. (pp. 665-691). Washington, DC, US: American Psychological Association. doi: 10.1037/14341-021

Blanton, H., \& Jaccard, J. (2006). Arbitrary metrics in Psychology. American Psychologist, 61(1), 27. doi: 10.1037/0003-066X.61.1.27

Brough, A. R., Wilkie, J. E., Ma, J., Isaac, M. S., \& Gal, D. (2016). Is eco-friendly unmanly? The green-feminine stereotype and its effect on sustainable consumption. Journal of Consumer Research, 43(4), 567-582. doi: $10.1093 /$ jcr/ucw044

Bruni, C. M., Chance, R. C., Schultz, P. W., \& Nolan, J. M. (2012). Natural connections: Bees sting and snakes bite, but they are still nature. Environment and Behavior, 44(2), 197-215. doi: $10.1177 / 0013916511402062$

Bruni, C. M., \& Schultz, P.W. (2010). Implicit beliefs about self and nature: Evidence from an IAT game. Journal of Environmental Psychology, 30(1), 95-102. doi: 10.1016/j.jenvp.2009.10.004

De Houwer, J., Teige-Mocigemba, S., Spruyt, A., \& Moors, A. (2009). Implicit measures: A normative analysis and review. Psychological Bulletin, 135(3), 347. doi: 10.1037/a0014211

Duffy, S., \& Verges, M. (2010). Forces of nature affect implicit connections with nature. Environment and Behavior, 42(6), 723-739. doi: $10.1177 / 0013916509338552$

Evans, G. (2005). A importância do ambiente físico. Psicologia USP, 16, 47-52. doi: 10.1590/S0103-65642005000100007

Ewert, A., \& Galloway, G. (2004). Expressed environmental attitudes and actual behavior: Exploring the concept of environmentally desirable responses. En International Outdoor Education Research Conference (pp. 1-8). Victoria, Australia: La Trobe University Bendigo. Recuperado de https://www.latrobe.edu.au/education/downloads/2004_ conference_ewert2.pdf

Gamberini, L., Spagnolli, A., Corradi, N., Sartori, G., Ghirardi, V., \& Jacucci, G. (2014). Combining implicit and explicit techniques to reveal social desirability bias in electricity conservation self-reports. Energy Efficiency, 7(6), 923-935. doi: 10.1007/s12053-014-9266-6

Gawronski, B., \& Bodenhausen, G. V.(2011). The associative-propositional evaluation model:Theory, evidence, and open questions. En M.P.Zanna \& J. M. Olson (Orgs.), Advances in experimental social Psychology (pp. 59-127). Amsterdam, The Netherlands: Elsevier. doi: 10.1016/ B978-0-12-385522-0.00002-0

Estudos de Psicologia, 24(3), julho a setembro de 2019, 292-304 
Gawronski, B., \& Hahn, A. (2017). Implicit measures: Procedures, use, and interpretation. En H. Blanton \& G. D. Webster (Orgs.), Foundations of social psychological measurement (pp. 29-55). Nueva York: Taylor \& Francis.

Geng, L., Liu, L., Xu, J., Zhou, K., \& Fang, Y. (2013). Can evaluative conditioning change implicit attitudes towards recycling?. Social Behavior and Personality: An International Journal, 41(6), 947-955. doi: 10.2224/sbp.2013.41.6.947

Geng, L., Xu, J., Ye, L., Zhou, W., \& Zhou, K. (2015). Connections with nature and environmental behaviors. PloS One, 10(5), 1-11. doi: 10.1371 /journal.pone.0127247

Gifford, R. (2014). Environmental psychology matters. Annual Review of Psychology, 65, 541-579. doi:10.1146/annurev-psych-010213-115048

Greenwald, A.G., \& Farnham, S.D. (2000). Using the implicit association test to measure self-esteem and self-concept. Journal of Personality and Social Psychology, 79(6), 1022. doi: 10.1037/0022-3514.79.6.1022

Greenwald, A. G., McGhee, D. E., \& Schwartz, J.L. K. (1998). Measuring individual differences in implicit cognition: the implicit association test. Journal of Personality and Social Psychology, 74 (6), 1464-1480. doi: 10.1037/0022-3514.74.6.1464

Greenwald, A. G., Poehlman, T. A., Uhlmann, E. L., \& Banaji, M. R. (2009). Understanding and using the implicit association test: III. Meta-analysis of predictive validity. Journal of Personality and Social Psychology, 97(1), 17-41. doi: 10.1037/a0015575

Günther, H. (2005). A Psicologia Ambiental no campo interdisciplinar de conhecimento. Psicologia USP, 16(1/2), 179-183. Recuperado de http://pepsic.bvsalud.org/scielo. php? script=sci_arttext\&pid=S1678-51772005000100019\&Ing= pt\&tIng $=p t$

Günther, H., \& Rozestraten, R. J. (1993). Psicologia ambiental: algumas considerações sobre sua área de pesquisa e ensino. Psicologia: Teoria e Pesquisa, 9(1), 109-124.

Janpol, H. L., \& Dilts, R. (2016). Does viewing documentary films affect environmental perceptions and behaviors?. Applied Environmental Education \& Communication, 15(1), 90-98. doi: 10.1080/1533015X.2016.1142197

Karpinski, A., \& Steinman, R. B. (2006). The single category Implicit Association Test as a measure of implicit social cognition. Journal of Personality and Social Psychology, 91, 16-32. doi: 10.1037/0022-3514.91.1.16

Klöckner, C. A. (2013). A comprehensive model of the psychology of environmental behavior: A meta-analysis. Global Environmental Change, 23(5), 1028-1038. doi: 10.1016/j.gloenvcha.2013.05.014

Levine, D. S., \& Strube, M. J. (2012). Environmental attitudes, knowledge, intentions and behaviors among college students. The Journal of Social Psychology, 152(3), 308-326. doi: 10.1080/00224545.2011.604363

Morren, M., \& Grinstein, A. (2016). Explaining environmental behavior across borders: A meta-analysis. Journal of Environmental Psychology, 47, 91-106. doi: 10.1016/j.jenvp.2016.05.003

Moser, G. (2003). La Psicología Ambiental en el siglo 21: el desafío del Desarrollo Sustentable. Revista de Psicología, 12(2), 11. doi: 10.5354/0719-0581.2012.17386

Nosek, B. A, Greenwald, A. G., \& Banaji, M. R. (2007). The Implicit Association Test at age 7: a methodological and conceptual review (pp. 265-292). En J. A. Bargh (ed.), Automatic processes in social thinking and behavior. Psychology press.
Olivos, P., \& Aragonés, J. I. (2013). Test de asociaciones implícitas con la naturaleza: Aplicación en España del "IAT-Nature". Revista de Psicología Social, 28(2), 237-245. doi: 10.1174/021347413806196672

Olson, M. A., \& Fazio, R. H. (2009). Implicit and explicit measures of attitudes: The perspective of the MODE model. En R. E. Petty, R. H. Fazio, \& P. Briñol (Orgs.), Attitudes: Insights from the New Implicit Measures (pp. 19-63). Nueva York, NY, US: Psychology Press

Payne, B. K., Burkley, M. A., \& Stokes, M. B. (2008). Why do implicit and explicit attitude tests diverge? The role of structural fit. Journal of Personality and Social Psychology, 94(1), 16. doi: 10.1037/0022-3514.94.1.16

Payne, B. K., Cheng, C. M., Govorun, O., \& Stewart, B. D. (2005). An inkblot for attitudes: affect misattribution as implicit measurement. Journal of Personality and Social Psychology, 89(3), 277. doi: 10.1037/0022-3514.89.3.277

Payne, B. K., \& Gawronski, B. (2010). A history of implicit social cognition: Where is it coming from? Where is it now? Where is it going? En B. Gawronski \& B. K. Payne (Orgs.), Handbook of implicit social cognition: Measurement, theory, and applications (pp. 1-15). Nueva York, NY, US: The Guilford Press.

Pinheiro, J. Q. (2003). Psicologia Ambiental brasileira no início do século XXI. Sustentável? En O. H. Yamamoto \& V. V. Gouveia (Orgs.), Construindo a Psicologia brasileira: desafios da ciência e da prática psicológica (pp. 279-313). São Paulo: Casa do Psicólogo

Ratliff, K. A., Howell, J. L., \& Redford, L. (2017). Attitudes toward the prototypical environmentalist predict environmentally friendly behavior. Journal of Environmental Psychology, 51, 132-140. doi: 10.1016/j. jenvp.2017.03.009

Rydell, R. J., \& McConnell, A. R. (2006). Understanding implicit and explicit attitude change: a systems of reasoning analysis. Journal of Personality and Social Psychology, 91(6), 995. doi: 10.1037/0022-3514.91.6.995

Sánchez-Miranda, M.P. (2016). La capacidad restaurativa de la naturaleza: En la búsqueda de su relación con las actitudes implícitas. Opción, 32(13), 840-863. Recuperado de http://produccioncientificaluz.org/ index.php/opcion/article/view/21627

Sánchez-Miranda, M. P., \& de la Garza González, A. (2015). Estudio del impacto de imágenes ambientales en los aspectos emocionales. Revista de Psicología (PUCP), 33(2), 387-410. Recuperado de http:// revistas.pucp.edu.pe/index.php/psicologia/article/view/13049/13662

Sánchez-Miranda, M. P., de la Garza González, A., \& Isolde Hedlefs, M. (2016). Implicit measures of environmental attitudes: A comparative study. International Journal of Psychological Research, 9(1), 40-51. doi: 10.21500/20112084.2099

Sánchez-Miranda, M. P., de la Garza González, A., \& Rangel Sandoval, M. L. (2013). Study about the emotional valence of environmental concepts using affective priming technique. International Journal of Psychological Research, 6(2), 50-58. doi: 10.21500/20112084.675

Sartori, G., Agosta, S., Zogmaister, C., Ferrara, S. D., \& Castiello, U. (2008). How to accurately detect autobiographical events. Psychological Science, 19(8), 772-780. doi: 10.1111/j.1467-9280.2008.02156.x

Schultz, P., Shriver, C., Tabanico, J. J., \& Khazian, A. M. (2004). Implicit connections with nature. Journal of Environmental Psychology, 24(1), 31-42. doi: 10.1016/S0272-4944(03)00022-7

Schultz, P., \& Tabanico, J. (2007). Self, identity, and the natural environment: Exploring implicit connections with Nature. Journal of Applied Social Psychology, 37(6), 1219-1247. doi:10.1111/j.1559-1816.2007.00210.x

Sriram, N., \& Greenwald, A. G. (2009). The Brief Implicit Association Test. Experimental Psychology, 56, 283-294. doi: 10.1027/1618-3169.56.4.283 
Actitudes implícitas en psicología ambiental. Una revisión de literatura

Stern, P.C. (2000). New environmental theories:Toward a coherent theory of environmentally significant behavior. Journal of Social Issues, 56(3), 407-424. doi: 10.1111/0022-4537.00175

Strack, F., \& Deutsch, R. (2004). Reflective and impulsive determinants of social behavior. Personality and Social Psychology Review, 3, 220-247. doi: 10.1207/s15327957pspr0803_1

Suchotzki, K., Verschuere, B., Van Bockstaele, B., Ben-Shakhar, G., \& Crombez, G. (2017). Lying takes time: A meta-analysis on reaction time measures of deception. Psychological Bulletin, 143(4), 428-453. doi: $10.1037 /$ bul0000087

Tate, K., Stewart, A. J., \& Daly, M. (2014). Influencing green behaviour through environmental goal priming: The mediating role of automatic evaluation. Journal of Environmental Psychology, 38, 225-232. doi: 10.1016/j.jenvp.2014.02.004

Thomas, G. O., \& Walker, I. (2016). The development and validation of an implicit measure based on biospheric values. Environment and Behavior, 48(5), 659-685. doi: 10.1177/0013916514553836

Truelove, H. B., Greenberg, M. R., \& Powers, C. W. (2014). Are implicit associations with nuclear energy related to policy support? Evidence from the brief implicit association test. Environment and Behavior, 46(7), 898-923. doi: 10.1177/0013916513480861

Turaga, R. M. R., Howarth, R. B., \& Borsuk, M. E. (2010). Pro-environmental behavior. Annals of the New York Academy of Sciences, 1185(1), 211-224. doi: 10.1111/j.1749-6632.2009.05163.x

Urrutia, G., \& Bonfill, X. (2013). The PRISMA statement: A step in the improvement of the publications of the Revista Española de Salud Pública. Revista Española de Salud Pública, 87(2), 99-102. doi: 10.4321/S1135-57272013000200001

Wang, X., Geng, L., Zhou, K., Ye, L., Ma, Y., \& Zhang, S. (2016). Mindfu learning can promote connectedness to nature: Implicit and explicit evidence. Consciousness and Cognition, 44, 1-7. doi: 10.1016/j. concog.2016.06.006

Wilson, T. D., Lindsey, S., \& Schooler, T. Y. (2000). A mode of dual attitudes. Psychological review, 107(1), 101-126. doi: 10.1037/0033-295X.107.1.101

Zhou, K., Ye, L., Geng, L., \& Xu, Q. (2015). How do implicit materialism and postmaterialism affect proenvironmental behavior?. Social Behavior and Personality: an international journal, 43(9), 1495-1505. doi: 10.2224/sbp.2015.43.9.1495

Jeremías David Tosi, Doctor en Psicología. Facultad de Psicología, Universidad Nacional de Mar del Plata/IPSIBAT, Becario Doctoral del Consejo Nacional de Investigaciones Científicas y Técnicas (CONICET). Docente de grado (Universidad Nacional de Mar del Plata). Dirección: Funes 3350, Mar del Plata (7600) Argentina. Tel.: +54 02234752526. Correo Eletronico: jeremiastosi@gmail.com

Ruben Daniel Ledesma, Doctor en Psicología. Facultad de Psicología, Universidad Nacional de Mar del Plata/IPSIBAT, Investigador del Consejo Nacional de Investigaciones Científicas y Técnicas (CONICET), Docente de grado (Universidad Nacional de Mar del Plata). Correo Eletronico: rdledesma@conicet.gob.ar

Ariane Kuhnen, Doutora em Ciências Humanas, Pós-Doutorado na UCDavis, University of California, Professora do Programa de Pós-graduação em Psicologia na Universidade Federal de Santa Catarina - UFSC. Correo Eletronico: arianekuhnen@gmail.com

Maíra Longhinotti Felippe, Doutora em Tecnologia da Arquitetura, Università degli Studi di Ferrara (UNIFE - Itália), Pós-doutorado em Psicologia, Universidade Federal de Santa Catarina (UFSC),

Pós-Doutorado em Arquitetura e Urbanismo, Universidade Federal de Santa Catarina (UFSC), Professora Adjunta no Departamento de Arquitetura e Urbanismo da Universidade Federal de Santa Catarina (UFSC). Correo Eletronico: m.l.felippe@ufsc.br

Recibido en 03.fev.18 Revisado en 14.ago.19 Aprobado en 16.out.19 